\title{
Acknowledgment to Reviewers of Children in 2020
}

\section{Children Editorial Office}

Citation: Children Editorial Office. Acknowledgment to Reviewers of Children in 2020. Children 2021, 8, 45. https://doi.org/10.3390/

children 8010045

Published: 14 January 2021

Publisher's Note: MDPI stays neutral with regard to jurisdictional claims in published maps and institutional affiliations.

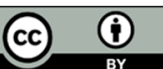

Copyright: $\odot 2021$ by the author. Licensee MDPI, Basel, Switzerland. This article is an open access article distributed under the terms and conditions of the Creative Commons Attribution (CC BY) license (http://creativecommons.org/licenses/by/4.0/).

MDPI AG, St. Alban-Anlage 66, 4052 Basel, Switzerland

Peer review is the driving force of journal development, and reviewers are gatekeepers who ensure that Children maintains its standards for the high quality of its published papers. Thanks to the cooperation of our reviewers, in 2020, the median time to first decision was 18 days and the median time to publication was 42 days. The editors would like to express their sincere gratitude to the following reviewers for their precious time and dedication, regardless of whether the papers were finally published:

Aadland, Eivind

Abalasei, Aurelia Beatrice

Abasse, Soumeth

Abecasis, Francisco

Agbangla, Nounagnon

Agoston, Anna Monica

Agrawal, Priyanka

Aguaded-Ramírez, Eva

Aguayo, Beatriz Berrios

Ahmad, Tanveer

Ahmed, Mohamed Lemine Cheikh Brahim

Akangire, Gangaram

Alaofe, Halimatou

Albadri, Sondos

Albright, Dana

Alinde, Keegstra

Allegaert, Karel

Alsharairi, Naser

Alsina, Miguel

Alves-Guerreiro, Jose

Ananieva, Elitsa

Andersson, Matthew A.

Angel, Herrera-Guerra

Antoniou, Panos

Antonogeorgos, George

Anzelewicz, Stefan Michał

Arany, Edith

Aritio-Solana, Rebeca

Arnbjörnsson, Einar

Arufe Giraldez, Victor

Aslanidis, Theodoros

Assari, Shervin

Astrid, Bertsche

Auten, Richard L.

Ávila-García, Manuel

Avtanski, Dimiter

Ayllón, Ester

Ayuso, Juan Mielgo

Bacchetta, Rosa
Bachou, Hanifa

Baddock, Sally A.

Bader, Peter

Bahmad, Hisham

Bahn, Geon Ho

Baía, Inês

Baile Ayensa, José Ignacio

Baj, Jacek

Bajwa, Amandeep

Baldwin, Heather J.

Balestrino, Roberta

Bang, Kyung-Sook

Barker, Alan

Barnes-Josiah, Debora

Bart, Orit

Bartkowski, John

Basset, David R.

Bassi, Carl J.

Bazylak, Grzegorz

Beauchaine, Theodore P.

Becker, Genevieve

Behrman, Andrea

Beinvogl, Beate

Bekteshi, Saranda

Bell, Laura

Bellato, Alessio

Belteki, Gusztav

Ben-Avie, Michael

Bender, Steven D.

Berbrayer, David

Bergstraesser, Eva

Berkers, Ruud

Berthon, Bronwyn

Bestman, Amy

Bielinis, Ernest

Bierens, Joost

Bignardi, Elio

Binder-Heschl, Corinna

Binford, Warren 


\author{
Bingham, Jenny \\ Biniwale, Manoj A. \\ Black, William Robert \\ Blackmore, Marie \\ Bláha, Ladislav \\ Boggero, Ian \\ Boles, Jessika C. \\ Bolton Moore, Carolyn \\ Bonam, Srinivasa Reddy \\ Bonilla, Diego A. \\ Booker, Cara \\ Borsani, Oscar \\ Borzì, Francesca \\ Bothe, Denise \\ Brand, Serge \\ Braun, David \\ Braun, Rudolf K. \\ Bremer, Andrew \\ Brewer, Hannah J. \\ Brinkman, Sally \\ Britt, Rodney D. \\ Bronikowski, Michał \\ Brottman, Gail \\ Brunelli, Sandro \\ Bryden, Pam \\ Bryndal, Aleksandra \\ Buckler, E. Jean \\ Bujak-Gizycka, Beata \\ Busjahn, Andreas \\ Byoung-Hee, Lee \\ Cadime, Irene \\ Cai, Demin \\ Calcagni, Giulio \\ Callister, Lynn Clark \\ Cameron, Noel \\ Campus, Guglielmo \\ Canciani, Elena \\ Capelli, Giovanni \\ Caridade, Sónia Maria Martins \\ Carli, Marco \\ Carmona-Mora, Paulina \\ Carpinelli, Luna \\ Carrouel, Florence \\ Carter, Bernie \\ Carter, Brian S \\ Casas, Rosa M. \\ Castillo, Silvia \\ Castillo-Rodríguez, Alfonso \\ Castro-Alonso, Cristina \\ Catto-Smith, Anthony \\ Cerník, David \\ Chalkley, Anna \\ Challagundla, Kishore \\ Champagne, Catherine \\ Chan, Chin-Kan \\ Chan, Ernest G. \\ Chan, Kak-Chen
}

Chandrasekharan, Praveen K.

Chawner, Samuel

Chen, Chao

Chen, Chiao-Nan

Chen, Chi-Nien

Chen, Gwo-Shing

Chen, Kong Y.

Chen, Yang

Chen, Yueh-Chih

Cheng, Haizi

Chiappedi, Matteo

Chien, Yin-Hsiu

Chillón Garzon, Palma

Ching, Christina

Chisari, Emanuele

Chiu, Hsiu-Ching

Chmelik, Frantisek

Choisne, Julie

Chou, Pang-Yun

Christian, Danielle

$\mathrm{Chu}$, Yen-Ju

Chuang, Hai-Hua

Chutiyami, Muhammad

Clark, Andrew

Clemente, Maria Grazia

Collins, Andrew B.

Collins, Christopher

Colombo-Dougovito, Andrew M.

Compagnon, Roxane

Conneely, Shannon E.

Constantinescu, Alexandru

Corbett, Blythe A.

Correll, Lynnie

Counil, Francois-Pierre

Coussens, Scott

Cozzani, Emanuele

Crane, Jeff $\mathrm{R}$.

Criss, Shaniece

Critser, Paul J.

Cross, Christina

Crowley, James

Crume, Peter K.

Cuenca, Alex

Cuesta Gómez, Alicia

Curtis, Jacqueline

Curto, Adrián

Cutrona, Carolyn

Czepczor-Bernat, Kamila

D'Amario, Maurizio

D'Souza, Genevieve

Dachsangvorn, Ona

Dalal, Priti G.

Dalimonte-Merckling, Danielle

Dall'Oglio, Luigi

Damtie, Debasu

Danysh, Heather E.

Das, Abhirup 
Davies, W. Hobart

Dawes, Colin

Dawes, Patrick

Day, Kaitlin

De Almeida Honório, Samuel Alexandre

De Gennaro, Luigi

De La Fuente-Solana, Emilia I.

De La Vega De Carranza, Rocío

De La Vega, Rocío

De Leeuw, Peter

De Müllenheim, Pierre-Yves

Deacy, Amanda

DeCocker, Katrien

Deeb, Wissam

Del Barba, Paolo

Del Pozo De La Calle, Susana

Delaney, Cassidy

Delaroche, Amy M.

Denis, Frederic

Deutsch, Nina

Devlin, Lori A.

Di Lorenzo, Mariana

Di Nardo, Matteo

Di, Sharon

Diogo, Elisete

Dixit, Anjali A.

Dixon, Andrew

Diykh, Mohammed

Dodero, Veronica

Dohme, Lea-Cathrin

Doi, Takehiko

Doll, Dietrich

Donohue, Lee T

Dorota, Gaskins

Dowell, Richard C.

Driessen, Geert

Dudeney, Joanne

Dudeney, Joanne E.

Dun, Matthew D.

Duncanson, Kerith

Dussel, Veronica

Dutra, Samia V.

Dynia, Jaclyn

Eaton, Simon

Edens, Cuoghi

Elce, Ausilia

Eliades, Theodore

Elias, Amanuel

Elisabetta, Cretella Lombardo

Elliott III, William

Engelhardt, Thomas E.

Esposito, Ciro

Esteban-Gorgojo, Ignacio

Evers, Patrick D.

Falsaperla, Raffaele

Fang, Di

Fasoli, Allison DiBianca
Favilli, Silvia

Feig, Emily

Feinstein, James

Feldkamp, Marcia

Feldman, Jacob I.

Felknor, Sarah A.

Fernández Carnero, Samuel

Fernandez, Mercedes

Ferrandiz, Carmen

Ferrara, Felicetto

Ferrara, Pietro

Ferrer-Sargues, Francisco José

Fiedorowicz, Ewa

Fink, James B.

Finley, Allen

Fiori, Cristian

Flores-Fraile, Javier

Floríndez, Lucía I.

Flynn, Emilee

Fonnes, Siv

Forget, Patrice

Forsum, Elisabet A.

Foulkes, Jonathon

Frader, Joel E

Freitas, Raquel

Friedman, Clive

Fusar-Poli, Laura

Gagnon, Joseph Calvin

Galan-Lopez, Pablo

Galeotti, Fabio

García-Galbis, Manuel Reig

García-Garnica, Marina

García-Muñoz Rodrigo, Fermin

García-Soidán, Jose L.

Garibotto, Giacomo

Garriboli, Massimo

Gatti, Flora

Gavini, Madhavi

Gawlowski, Pawel

Gaze, Mark

Gelzo, Monica

Geoffray, Marie Maude

George, Koumantakis

Gepner, Yftach

Gerson, Trevor

Giacani, Lorenzo

Gibson, Philippa

Gien, Jason

Gierczyk, Marcin

Gigante, Antonietta

Giles, Audrey

Giontella, Alice

Glenister, Kristen

Godoy-Izquierdo, Débora

Goggin, Maureen Daly

Gomes, Thayse Natacha

Gómez-Baya, Diego 
Gómez-de-Terreros-Guardiola, Montserrat

Gómez-Tafalla, Ana

González Azpeitia, Gloria

González, Daniel

Gonzalez-Alonso, Fernando

Gonzalez-Jurado, Jose Antonio

Goodwin, Michaela

Gordish-Dressman, Heather

Gori, Davide

Gorodzinsky, Ayala

Gosav, Evelina Maria

Goudas, Marios

Gounaris, Antonios K.

Graham, Tiffany

Greenhalgh, David

Gribble, Karleen

Grillo, Andrea

Groba, Betania

$\mathrm{Gu}$, Xiangli

Guevara, Jennifer

Guinsburg, Ruth

Gupta, Medhavi

Gustafsson, Nina-Katri

Gutíerrez Hervas, Ana Isabel

Guynes, Kristen

Gwynette, McLeod

Haapa, Toni

Hakeem, Faisal F.

Halley, Meghan

Halski, Tomasz

Han, Sungil

Hanke, Wojciek

Hanna, Amgad

Haro, Gonzalo

Harrington, Deirdre M.

Hart, Lara

Harton, Anna

Hausser, Ingrid

Heaton, Steven M.

Hegyi, Thomas Hegyi

Heinen, Mirjam

Herbert, Anthony

Herbuela, Von Ralph Dane M.

Hernandez Fernandez, Antonio

Hernandez, Erika

Hernández-Alonso, Pablo

Herrero, Oscar

Herzog, Sarah

Hida, Azumi

Hidalgo Lopezosa, Pedro

Hillman, Heidi

Hirsch, Christian

Ho, Pei-Shan

Hoellwarth, Jason

Hojan, Katarzyna

Holt, Kelly

Homma, Tetsuya
Hong, Bo Young

Hopp, Russell

Horhat, Delia

Howerton-Fox, Amanda

Hsu, Li-Chi

Huang, Charles

Hughes, Christopher V.

Hughes, Jean R.

Hügle, Boris

Hugrass, Laila

Huisman, Sylvia

Hulteen, Ryan

Hurtado, Ghaffar Ali

Husain, Ralf

Hyun, Taisun

ILAND, HARRY

Ilyas, Mohammed

Ines, Van Keer

Ing, Frank F

Innerd, Alison

Iorio, Raffaele

Ishii, Yumiko

Iskusnykh, Igor Y.

Izzo, Riccardo

Jabłonowski, Zbigniew

Jacob, Jenna

Jácome, Cristina

Jacques, Angela

Jaehne, Anja Kathrin

Jälevik, Birgitta

Jancelewicz, Tim

Janowski, Miroslaw

Janssens, Thomas

Januszkiewicz-Lewandowska, Danuta

Jasien, Joan

Jasiewicz, Barbara

Jayaprakash, Priyamvada

Jenkins, Todd M.

Jillella, Dinesh

Jones, Paul C.

Jones, Rachel

Joronen, Katja

Jose, Perez

Juárez-Ruiz De Mier, Rocío

Jungquist, Carla R.

Jurjans, Kristaps

Kaczyńska, Katarzyna

Kaditis, Athanasios

Kaiser, Pamela

Kaissi, Ali Al

Kaminska, Margaret

Kampouras, Asterios

Kan, Pui Fong

Kanakri, Shireen

Kane, Kyra

Kang, Augustine W.

Kao, Grace 
Karasik, Lana B.

Karisola, Piia

Kasper-Jędrzejewska, Martyna

Katherine, Prowse

Katz, Phillip

Katz, Terry

Kawamura, Tomoyuki

Keil, Margaret F.

Keisaku, Sato

Kendall-Taylor, Nathaniel

Kent, Jacqueline

Kent, Jennifer

Kesavan, Kalpashri

Kevin, Ackermans

Khalil, Nadim

Khanday, Mudasir A.

Khubchandani, Jagdish

Kim, Hyungjoo

Kim, Jiyong

Kim, Sang-Dol

King, Christopher D.

Knisel, Elke

Kobel, Susanne

Koechlin, Helen

Kolodziejski, Pawel Antoni

Korcz, Agata

Korinthenberg, Rudolf

Kosanovic, Djuro

Kotzalidis, Giorgio D.

Koutelidakis, Antonios E.

Kovacic, Katja K

Kozakiewicz, Marcin

Kozioł-Kozakowska, Agnieszka

Kozlik-Feldmann, Rainer Gerhard

Krajnak, Kristine M.

Kristen (Kris), Dovgan

Krüger, Björn

Krupp, Lauren B.

Kumar, Kishore Raj

Kumar, Maya M.

Kumar, Suneel

Kuo, Hsien-Wen

Kuppusamy, Maniselvan

Kurata, Mie

Kurosaka, Hiroshi

Kushner, Brian H

Kyvelidou, Anastasia

L. Wood, Megan

La Mantia, Ignazio

Lăcătușu, Cristina-Mihaela

Lack, Sharon

Lademann, Hanne

Lamba, Vineet

Lander, Natalie

Landis, Carolyn E.

Landt, Eskild

Lane, Ginny
Lane, Shelly J.

Lange, Joanna

Lanks, Charles W.

Lara Sánchez, Amador Jesús

Laudanska-Krzeminska, Ida

Lawson, Nicholas D.

Lebon, Sébastien

Lee, Hui Jai

Lee, Jason Jiunshiou

Lee, Joonyoung

Lee, Jung Eun

Lee, Justin

Lee, YoonJung

Lefere, Sander

Lemanek, Kathleen

León-Quismondo, Jairo

Lera, María José

Lerma, Claudia

Leva, Ernesto

Levine, Shel

Li, Chia-Jung

Li, Dian-Jeng

Li, Shuai

Li, Tengfei

Lichenstein, Richard

Lin, Hui-Chu

Lin, Yuan-Chi

Lin, Yuh-Jyh

Lin, Yung-Chieh

Llopis-González, Agustín

Lober, Angela

Locher, Cosima

Lombardi, Marco

Lombardi, Tommaso

Longobardi, Claudio

Lonhart, Julia A.

Lopez, Antonella

Lorås, Håvard

Lourenço, Olga

Lucchese, Alessandra

Luis Ubago, Jose

Lukowski, Angela F.

Luna, Ruth Ann

Lund, Jonathan

Luque Moreno, Carlos

Łuszczki, Edyta

Lysecki, Dave

Ma, Sihui

Maatouk, Ismael

Maclellan, Reid A.

MacLeod, Stuart

MacMillan, Freya

Mahran, Amr

Mair, Jacqueline

Makanji, Rikesh

Malik, Tariq

Malin, Katrina 
Małysz-Cymborska, Izabela

Manali, Effrosyni

Mancinelli, Elisa

Manco, Licínio

Mandelbaum, David

Mannion, Arlene

Manolopoulos, Anna

Mantziari, Anastasia

Marcão, Ana

Marchesi, Alessandra

Marlu, Raphaël

Marshall, Teresa A.

Martín Valero, Rocío

Martin, Erin I.

Martin, Ryan

Martin, Sarah

Martina, Sandoná

Martinez Selva, Dawid

Martinez-Carrión, José-Miguel

Martins, Ian

Martinuzzi, Andrea

Mascherini, Gabriele

Masetti, Riccardo

Mason, Carolynne

Maspero, Cinzia

Mathew, Mona

Mathew, Rajamma

Mattsson, Janet

Matziou, Vasiliki

Mbogori, Teresia

McCartan, Claire

McCarthy, Kevin

McClafferty, Hilary

McClain, Kenneth L.

McConnell, Patrick I.

McDowell, Michael

McLafferty, Sara

Mecías-Calvo, Marcos

Meddings, David

Mei-heng, Lin

Mena-Tudela, Desirée

Menon, Renuka T.

Mentis, Alexios Fotios A.

Merks, Piotr

Merquiol, Fanette

Merrick, Jillian S.

Mester, Alexandru

Miçooğulları, Bülent Okan

Middleton, Mark

Migita, Ohsuke

Mikhael, Michel

Mileti, Ilaria

Miller, Jillian Vinall

Minotti, Bruno

Mitchell, Wendy G.

Mitra, Joy

Miyagi, Hisayuki
Miyamura, Takako

Miyoshi, Takekazu

Mize, Minnie

Mocny-Pachońska, Katarzyna

Moding, Kameron

Molcho, Michal

Molitor, Sabine

Monaro, Merylin

Mondanaro, John

Mongkuo, Maurice Y.

Monsarrat, Paul

Montanino, Mario

Montemurro, Nicola

Moore, Kristin

Morabito, Antonino

Moral-García, José Enrique

Morand-Beaulieu, Simon

Morasiewicz, Piotr

Morgan, Jill

Morgan, Kelly

Mori, Masaaki

Morris, Joan

Moses, Jonathan

Motamed, Cyrus

Moutelíková, Romana

Muego, Melissa

Muiesan, Maria Lorenza

Mukherjee, Shibabrata

Mulcahy Levy, Jean M.

Muñoz, Cynthia E.

Munoz, Flor M.

Muñoz-López, Francisco

Myers, Samuel

Nahm, Francis Sahngun

Nair, Jayasree

Najafi, Nadia

Nakata, Yoshio

Nam, Ok Hyung

Namba, Fumihiko

Nandalike, Kiran

Nandi, Deipanjan

Narkewicz, Michael R.

Natarajan, Sathish Kumar

Nath, Audrey

Nelson, Sarah M.

Nemeș, Roxana Maria

Neves, Rui

Newman, Christopher

Nguyen, Hoang $\mathrm{H}$.

Nguyen, Thinh

Nicholson, Maribeth

Nieminen, Pentti

Niermeyer, Susan

Njoku, Anuli

Noble, Fiona

Nordström, Thomas

Nota, Alessandro 
Notas, George

Nouri, Aria

Noviello, Carmine

Nowak, Paweł Fryderyk

Nsabimana, Epaphrodite

Nucera, Riccardo

Nwabudike, Lawrence Chukwudi

Oberle, Megan

Oei, Ju Lee

Ohliger, Shelley

O'Leary, Fenton

Oledzka, Magdalena

Oliva, Antonio

Oliván-Gonzalvo, G.

Oliva-Pascual-Vaca, Jesús

Oppici, Luca

Osooli, Mehdi

Otsuka, Mitsuo

Ouellet, Marilou

Owens, Matt

Owili, Patrick Opiyo

Ozanne-Smith, Joan

Ozbič, Martina

Paap, Kenneth R.

Pacini, Matteo

Paczkowska-Abdulsalam, Magdalena

Padmanabhan, Pradeep

Palakurthi, Bhavana

Pan, Yijun

Panaitescu, Anca

Pangrazzi, Luca

Papadopoulou, Marianna

Papakonstantinou, Evgenia

Papamitsou, Theodora

Paprocka, Justyna

Park, Eun-Young

Park, Jusik

Park, Sook-Hyun

Parker, Alasdair Pj.

Parodi, Aurora

Pasman, Wilrike J.

Paterson, Rebecca S.

Patiño, María José Martínez

Patro-Małysza, Jolanta

Paweł, Tomaszewski

Pearl, Phillip

Pearn, John

Peila, Chiara

Peng, Mei

Perdikaris, Pantelis

Pereira, Armanda

Peressotti, Francesca

Pérez-Bermejo, Marcelino

Pérez-Fuentes, María Del Carmen

Perin, Cecilia

Perry, Sarah E.

Pesavento, Russell
Petca, Rǎzvan

Peter, Metcalfe

Peterson, Catherine

Petousi, Vasiliki

Peyer, Karissa

Philip, Roy K.

Piątkowska, Monika

Pickett, Andrew C.

Picoraro, Joseph A.

Piersigilli, Fiammetta

Pinheiro, Fabio

Pinheiro, Joaquim

Pitti, Julio Alvarez

Plevinsky, Jill

Pogorelić, Zenon

Pokorska-Niewiada, Kamila

Pop, Tudor Lucian

Popiołek-Kalisz, Joanna

Poulimeneas, Dimitris

Prasad, Amit A.

Preez, Surita Du

Próchniak, Piotr

Procino, Giuseppe

Proctor, Mark R.

Prosperi, Margherita

Przybyła-Basista, Hanna

Pulido, Olga

Qadri, Ghalib

Qvist, Niels

Racil, Ghazi

Radnor, John M.

Radoja, Ivan

Ramamurthi, Ram

Ramirez-Graniz, Irwin Andrés

Ramiro-Cortijo, David

Ranabhat, Chhabi

Ranjith, Kamity

Rapkin, Louis

Rascher, Wolfgang

Raveendran, Sanjeetha

Rawat, Munmun

Rehman, Laurene

Rehman, Michael

Reis Gaya, Anelise

Reiss, Allan L.

Renate, Fuiko

Resiere, Dabor

Reula, Ana

Richards, Noni

Richardson, Patricia A.

Riedel, Thomas

Riley, Nicholas

Rima, Samy

Roberts, Michael W.

Robertson, Scott

Robinson, Sonia

Rodrigues, Carina 
Rodríguez-Almagro, Daniel

Rolle, Udo

Romano, Alberto

Romeo, Deborah

Romeo, Domenico

Roscoe, Clare

Rosi, Eleonora

Ross, Jennifer

Ross, Julie

Rothe, Thomas

Rothschild, Bruce

Rozensztrauch, Anna

Rozerta, Sokou

Rudolph, Henriette

Rustico, Lorenzo

Rusy, Lynn M.

Ryu, Ji-won

Sabbagh, Charles

Sacchi, Chiara

Sakamoto, Ryuichi

Sakhnini, Victoria

Salamon, Katherine S.

Salö, Martin

Saltaji, Humam

Salvador-Coloma, Pablo

Samadi, Sayyed Ali

Samppa, Ryhanen

Sánchez-Miguel, Pedro Antonio

Sánchez-Sánchez, Eduardo

Sánchez-Zafra, María

Sanchis-Soler, Gema

Sanfi, Ilan

Sani, Ana

Sankararaman, Senthilkumar

Sankarasubramanian, Vishwanath

Sansone, Francesco

Santos, Maria Paula

Santos, Sofia

Santos-Lozano, Alejandro

Sar, Bibhuti K.

Saraiva, Linda

Sathish, Thirunavukkarasu

Satoh, Taroh

Savant, Adrienne

Scala, Melissa

Scharoun Benson, Sara

Schechter, Neil

Schechtman, Samuel

Scherzer, Daniel J.

Schlegel, Peter N.

Schmidt, Andrew

Schmidt, Sabrina Krogh

Schmitt-Wilson, Sarah

Schroeder, Krista

Scrimshire, Ashley

Scurati, Raffaele

Sederstrom, Nneka
Serbic, Danijela

Serra-Paya, Noemi

Severino, Paolo

Sfeatcu, Ionela Ruxandra

Shah, Rugmini

Shahmoradi, Mahdi

Shamshirsaz, Alireza A.

Shankland, Rebecca

Sharma, Adhikarimayum Lakhikumar

Sharma, Atul

Sharma, Sapna

Sharma, Vinay

Sharmin, Sonia

Sharon, Denise

Sherrington, Anna Maria

Shiao, Chih-Chung

Shim, Jung Ok

Shin, Donghee

Shindova, Maria

Shinnar, Shlomo

Shinsugi, Chisa

Shkodra, Morena

Shulman, Stanford T

Siafis, Spyridon

Siegel, Robert M.

Silva, Maria-Raquel G.

Silvia, Paul

Simioni, Carolina

Simone, Pisano

Singh, Ajay Pratap

Singh, Rajbir

Sinnott, Catherine J.

Sisk, Cara

Siziba, Linda

Skinner, Christopher

Slee, Philllip

Sleet, David

Slezak, Ryszard

Slusher, Tina M.

Smith Roley, Susanne

Smith, Allison

Snyder, Julie

Sobey, Jenna

Sofo, Seidu

Sommer, Grit

Song, In Gyu

Sourial, Maryanne Y.

Spivakovsky, Silvia

Sprague, Wendy

Sreedher, Gayathri

Sribnick, Eric A.

Staderini, Edoardo

Stalsberg, Ragna

Staunstrup, Nicklas Heine

Stavinoha, Peter L.

Stefani, Laura

Stewart, Thomas 
Stiver, Corey

Stokes, Lynissa

Stone, Amanda

Straburzynski, Marcin

Strunecka, Anna

$\mathrm{Su}$, Erik

Sun, Naiyi

Surendran, Sushitha

Svoboda, Laurie

Sweeney, Brooke

Tagg, Andrew

Taked, Takayuki

Tamir, Sharon Ovnat

Tammelin, Tuija

Tang, Jingjing

Tanoubi, Issam

Tarraga-Minguez, Raul

Taylor, Danielle H.

Taylor, Greig

Te Nijenhuis, Jan

Teilmann, Grete Katrine

Teng, Ru-Jeng

Tennant, Marc

Teran-Garcia, Margarita

Thavamani, Aravind

Theorell-Haglöw, Jenny

Thielen, Beth

Thomas, Pauline A.

Thompson, Deborah

Tichko, Parker

Tilga, Henri

Timmers, Inge

Timpani, Cara

Ting, Chien-Kun

To, Yasuo

Toczylowski, Kacper

Toida, Chiaki

Tolaymat, Abdullah

Tomczak, Andrzej

Tomczyk, Łukasz

Tong, Yat-Ching

Tóth, Ákos

Toyoda, Hidemi

Traczyk, Iwona

Trahair, Toby

Trajanoska, Katerina

Trakada, Georgia

Tran, Susan T.

Trip, Simona

Tripathi, Jitendra Kumar

Triunfo, Stefania

Truong, Uyen

Tryggestad, Jeanie

Tsimpli, Ianthi Maria

Tsytsarev, Vassiliy

Turkmen, Mutlu

Umpaichitra, Vatcharapan
Urv, Tiina K.

Valitutti, Francesco

Van Arendonk, Kyle J.

Van Baar, Anneloes

Van Bergen, Christiaan

Van Der Helm, Peer

Van Der Mark, Elise

Van Dijk, Monique

Van Kann, Dave

Van Kann, Dave (D.H.H.)

Van Tilburg, Miranda A.L.

Vanderloo, Leigh $M$.

Vatsayan, Anant

Veazey, Leah Williams

Vemuri, Ravichandra

Verrastro, Valeria

Vesoulis, Zachary

Vidaurri, Lytorre

Vieira, Margarida

Villani, Ciro

Virella, Daniel

Vittori, Alessandro

Volochayev, Rita

Von Döbeln, Ulrika

Von Heideken, Johan

Voss, Megan E.

Vuille-dit-Bille, Raphael Nicolas

Wager, Julia

Wagner, Jessyca

Wahl-Alexander, Zachary

Walsh, Shana

Walsh, Stephanie M.

Walters, Camila B.

Wang, Alberta

Wang, Cynthia

Wang, Jianxiong

Wang, Lei

Wang, Tang-Chuan

Ward, Kenneth D.

Warner, John O.

Warschburger, Petra

Wasilewska, Eliza

Wasniewska, Malgorzata

Watanabe, Jun

Watanabe, Kenichiro

Watson, Michael

Wawrzyniak, Agata

Weingarten, Kevin

Weledji, Elroy Patrick

Weres, Aneta

Weyand, Angela C.

Whitaker, Amanda T.

White, Hank

White-Lewis, Sharon

Wiart, Lesley

Wickström, Ronny

Wijesinghe, Ruchire Eranga 
Williams, Alison

Williams, Cydni N.

Williams, Marc

Williams, Sara

Williams, Sara E.

Williamson, Paul

Wilson, Jenny L.

Withrow, Nicole

Włodarek, Dariusz

Wojciechowski, Wally

Wong, William

Wood, Thomas R.

Woods-Townsend, Kathryn

$\mathrm{Wu}$, Chiao-En

$\mathrm{Wu}, \mathrm{Zhi}-\mathrm{Fu}$

Xie, Zhiqun

$\mathrm{Xu}$, Furong

Yamauchi, Melissa S.W.

Yanai, Kazuhiko

Yang, Hui-Ting

Yang, Jimin
Yang, Shin-seung

Yarger, Heather

Yen, Cheng-Chieh

Yen, Cheng-Fang

Yokomichi, Hiroshi

Yousefzadeh, Sepideh

$\mathrm{Yu}$, Hong-Ren

Zadra, Cinzia

Zagalaz Sánchez, María Luisa

Zaghloul, Nahla

Zakrocka, Izabela

Zaman, Widaad

Zambrano, Rodrigo Elías

Zanotto, Lucia

Zarantonello, Paola

Zernikow, Boris

Zhang, Yinfeng

Zhao, Baoyin

Zheng, Quan Toh

Zolnikov, Tara Rava

Zotti, Francesca 